\section{Rehabilitation of hemianopic dyslexia: no words necessary}

Rehabilitation of hemianopic dyslexia-an impairment of reading ability caused by loss of part of the visual field-can be achieved by compensatory oculomotor training. This training method uses text-based materials, but whether text is essential to the rehabilitation technique is unclear. A UK study has now shown that training with nontext material (specifically, Arabic numerals) is as effective as text-based training for the rehabilitation of hemianopic dyslexia.

Schuett et al. recruited 40 patients with homonymous parafoveal visual field loss and impaired reading ability, who were divided into two equal groups, one of which used words as the basis for relearning eye-movement control while the other used Arabic numerals. Both methods were found to be equally effective for improving reading performance and the eye movements associated with reading. However, practicing eye movements with numerals was reported to be less distressing, tiring and frustrating for patients than word-based practicethe authors note that the reading task itself frequently seemed to cause distress, and also point out that numeral-based training removes patients' tendency to guess at the completion of words before they fully enter the visual field. All patients reported satisfaction with the outcome of treatment.

Although both rehabilitation techniques were successful in improving reading ability, neither had any effect on patients' visual exploration performance. The authors suggest that these two abilities may be controlled by different yet overlapping neural networks and, therefore, require specific treatment for their improvement.

Original article Schuett S et al. (2008) Rehabilitation of hemianopic dyslexia: are words necessary for re-learning oculomotor control? Brain 131: 3156-3168

\section{Reduction of L-DOPA-induced dyskinesia in animal models of Parkinson disease}

Previous studies in a rat model of Parkinson disease $(P D)$ have shown that dyskinesia induced by L-DOPA - a treatment-limiting adverse effect of long-term administration-is caused by the release of dopamine from 5-hydroxytryptamine
(5-HT) neurons. In a follow-up study, Muñoz et al. investigated the effect of 5-HT-receptor blockade in macaque and rat models of PD with L-DOPA-induced dyskinesia.

Administration of a $5-\mathrm{HT}_{1 \mathrm{~A}}$-receptor agonist to six monkeys with pharmacologically induced PD symptoms and dyskinesia produced a dosedependent reduction in dyskinesia without reducing the antiparkinsonian effect of L-DOPA. A 5- $\mathrm{HT}_{1 \mathrm{~B}}$-receptor agonist, however, did not reduce $L-D O P A$-induced dyskinesia. When the two agonists were administered together at low doses, the $5-\mathrm{HT}_{1 \mathrm{~B}}$-receptor agonist potentiated the effect of the $5-\mathrm{HT}_{1 \mathrm{~A}}$-receptor agonist, and reduced dyskinesia by $80 \%$ without affecting the therapeutic activity of L-DOPA. High doses of the combined agonists also produced this synergistic effect but reduced the antiparkinsonian effect of L-DOPA.

The authors then tested prolonged administration of $5-\mathrm{HT}_{1}$-receptor agonists in 140 rats with 6-hydroxydopamine-induced lesions that were receiving daily L-DOPA. Coadministration of the $5-\mathrm{HT}_{1}$-receptor agonists once a day for 2 weeks prevented dyskinesia and the upregulation of FosB in striatal neurons-a marker of the cellular effects that underlie L-DOPAinduced dyskinesia-without affecting the therapeutic properties of L-DOPA. Once 5-HTreceptor agonist treatment was halted, rats remained protected from dyskinesia for at least 2 weeks.

The authors suggest that simultaneous pharmacological activation of $5-\mathrm{HT}_{1 \mathrm{~A}}$ and 5$\mathrm{HT}_{1 \mathrm{~B}}$ receptors might provide a therapeutic benefit in patients with PD undergoing long-term L-DOPA treatment.

Original article Muñoz A et al. (2008) Combined 5- $\mathrm{HT}_{1 \mathrm{~A}}$ and $5-\mathrm{HT}_{1 \mathrm{~B}}$ receptor agonists for the treatment of L-DOPAinduced dyskinesia. Brain 131: 3380-3394

\section{Ratio of tau forms in the CSF identifies patients with progressive supranuclear palsy}

Borroni et al. have previously demonstrated that both extended $(55 \mathrm{kDa})$ and truncated $(33 \mathrm{kDa})$ forms of tau protein are present in the cerebrospinal fluid (CSF) and cerebral cortex of patients with progressive supranuclear palsy (PSP). This group has now shown that a low ratio of these tau forms reliably differentiates patients 